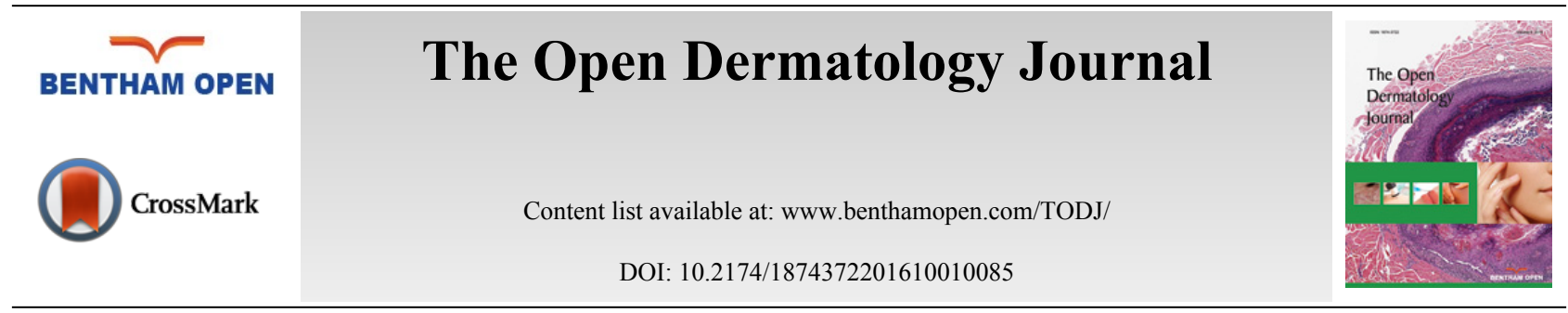

REVIEW ARTICLE

\title{
Topical Antiacne Drugs Delivery Systems
}

\author{
Tesfaye Gabriel ${ }^{*}$
}

Department of Pharmaceutics and Social Pharmacy, School of Pharmacy, College of Health Sciences, Addis Ababa University, P. O. Box 1176, Addis Ababa, Ethiopia

Received: August 23, 2016

Revised: September 10, 2016

Accepted: October 03, 2016

\section{Abstract: \\ Background:}

Acne vulgaris (commonly called acne) is the most prevalent skin complication of different causes with a higher prevalence in adolescents. Topical administration is used as first-choice therapy in mild acne, whereas for moderate and severe acne, systemic administration is required in addition to topical therapy. Mechanisms by which treatments act are: normalizing shedding into the pore to prevent obstruction, destruction of P.acnes, suppression of inflammation, and hormonal management.

\section{Objective:}

This review focuses on the novel drug delivery systems displaying a strong ground for topical treatment of acne in order to enhance the therapeutic performance of the topical antiacne agents with improved patience compliance and a concomitant reduction in the side effects.

\section{Method:}

This literature review was obtained from electronic search on Pubmed, Google Scholars, Researchgate, Scimago, CABI, DOAJ, CiteFactor, GLOBAL HEALTH, Universal Impact Factor, Hinari among many others and also search was conducted on individual journals and manuals.

\section{Conclusion:}

Amongst various novel drug delivery systems, vesicular carriers like liposomes and niosomes, micro sponges, microemulsions, solid lipid nanoparticles, hydrogels, emulsifier-free formulations, fullerenes and aerosol foams have been reported as novel topical administration of antiacne drugs. Liposomes have been extensively explored and their ability to optimize and improve topical therapy has been proved by several clinical trials. Microemulsions, microsponges, solid lipid nanoparticles and hydrogels also exhibit a tremendous potential for commercialization.

Keywords: Anti-inflammatory effects, Hormonal manipulation, Hydrogels, Liposomes and niosomes, Micro sponges, Microemulsions, P. acnes, Solid lipid nanoparticles, Topical therapy.

\section{INTRODUCTION}

Skin is considered to be the largest organ consisting of three layers: the epidermis, the dermis, and the hypodermis [1]. The most outer non-living part of the epidermis, the stratum corneum (SC), is the key diffusional resistance to percutaneous absorption. The exceptional resistance properties of the SC can be ascribed to its unique structure and composition. The viable epidermis is situated beneath the SC and responsible for the generation of the SC. The dermis is directly adjacent to the epidermis and composed of a matrix of connective tissue, which renders the skin its elasticity

\footnotetext{
* Address correspondence to this author at the Department of Pharmaceutics and Social Pharmacy, School of Pharmacy, College of Health Sciences, Addis Ababa University, P. O. Box 1176, Addis Ababa, Ethiopia; Tel: +251 912049154; E-mails: tesfu.gabriel@gmail.com, tesfaye.gabriel@aau.edu.et
} 
and resistance to deformation $[2,3]$.

The outer layer of the skin forms an effective barrier to retain water within the body and keep exogenous compounds out of the body. As a result, the major problem in dermal and transdermal drug deliveries is the low penetration of drug compounds through the SC. Dermal drug delivery comprises the topical application of drugs for the local treatment of skin diseases. It requires the permeation of a drug through the outer skin layers to reach its site of action within the skin, with little or no systemic uptake. The application of drugs to the skin for systemic therapy is referred to as transdermal drug delivery. Hence, it is required that a pharmacologically potent drug reaches the dermis where it can be taken up by the systemic blood circulation. In either case, the drug has to cross the outermost layer of the skin, the SC [1].

\subsection{The Viable Epidermis}

The layers in the epidermis are: stratum basale, stratum spinosum, stratum granulosum, and SC [4]. The innermost layer of the epidermis, the stratum basale, consists of a single layer of columnar-shaped, undifferentiated stem cells. As the cells produced by the basal layer move upward, they alter morphologically as well as histochemically to form the outermost layer, the SC. Over a 4 to 5-week period the entire epidermis is renewed [5].

\subsection{The SC}

$\mathrm{SC}$ is considered to be the major diffusional resistance for different materials like water [2, 3]. It is about 10 to $20 \mathrm{~A}$ $\mathrm{m}$ in thickness when dehydrated but swells to several times this thickness when entirely humid [6]. It comprises 10 to 25 layers parallel to the corneocytes, as protein bricks embedded in a lipid mortar [7]. During the changeover of the mature keratinocyte into the corneocyte, profilaggrin that is released from the keratohyalin granules is dephosphorylated and transformed to filaggrin monomers. Filaggrin is converted into free amino acids and other derivatives, which contribute to the wetness of the SC [8].

\subsection{Permeation Route Across the SC}

Compounds could cross skin in two mechanisms: the transappendageal path and the transepidermal path. The transappendageal pathway is believed to be of less importance than the transepidermal route because of its relatively small area, approximately $0.1 \%$ of the total skin area [8]. However, some findings have revealed the possibility of specifically targeting certain compounds to the pilosebaceous structures $[9,10]$. The success rate is mainly dependent on the lipophilicity of the permeant and the composition of the vehicle [11]. Due to the highly impermeable property of the cornified envelope, the tortuous intercellular route is proposed to be the key route for majority drug entities [12].

\section{ACNE VULGARIS}

The term acne is derived from the Greek $\alpha \dot{\kappa} \mu \dot{\eta}$ refering to the occurrence of pustules and papules. Acne vulgaris (commonly called acne) is the most prevalent skin complication of different causes with a higher prevalence in adolescents. The majority of the acne sufferers exhibit mild to moderate acne initially, which progresses to the severe form in certain cases [13, 14]. For most people, acne diminishes over time and tends to disappear. However, some individuals will continue to suffer well into their thirties, forties and beyond [15]. The face and upper neck are most commonly influenced, but the chest, back and shoulders could possess acne too. Aside from scarring, its main effects are psychological, such as reduced self-esteem and depression or suicide [16].

\subsection{Causes of Acne}

Acne typically results from blockages in follicles. Enlargement of sebaceous glands and an increase in sebum production occur with increased androgen (DHEA-S) production at adrenarche. The microcomedo may enlarge to form an open comedo (blackhead) or closed comedo (whitehead). Whiteheads are the direct result of skin pores becoming clogged with sebum, naturally occurring oil, and dead skin cells. Propionibacterium acnes can cause inflammation, leading to inflammatory lesions (papules, infected pustules, or nodules) in the dermis around the microcomedo or comedo, which results in redness and may result in scarring or hyperpigmentation [17].

The main cause of acne is not well known. Notwithstanding, there are numerous related factors reported: hereditary, hormonal activity, for example, menstrual cycles and puberty, inflammation, skin irritation, anxiety, through expanded yield of hormones from the adrenal (anxiety) organs with some questions, hyperactive sebaceous organs, auxiliary to the hormone sources, amassing of dead skin cells, microbes in the pores, utilization of anabolic steroids, any drug 
comprising lithium, barbiturates or androgens, presentation to certain concoction mixes, for example, chlorinated dioxins and halogens and frequent use of amphetamines [18 - 20].

\subsection{The Physical Effects of Acne}

The impacts of acne - both physical and emotional - can last much longer than your breakouts. Indeed, even after sores have healed, they can leave lasting reminders. It's hard to avoid scars of acne. However,acne scars can be treated. Post-inflammatory hyperpigmentation and macules can be tackled with bleaching agent. Some superficial skin inflammation scarring can be treated with topical resurfacing agents, similar to Retinol, which is accessible in numerous over-the-counter preparation, and additionally in prescription only medicines, for example, Retin-A and Renova. Different types of scarring can be enhanced with microdermabrasion (at least 6-8 medications are normally required) or dermatologic surgery. It may not be conceivable to reestablish your skin to its pre-acne appearance - however in the event that your scars significantly affect your well-being, it merits considering [21].

\section{TREATMENTS}

Topical treatment is usually utilized as first-choice therapy in minor acne, though for severe and complicated acne, systemic treatment is vital along with topical treatment. However, it was reported that topical intralesional triamcinolone and lincomycin combination was effective to eliminate severe of nodulocystic lesions of acne. Currently, so many topical medications are accessible that influence the primary pathogenetic factors. Though topical treatment has an essential place in acne management, adverse reactions connected with different topical antiacne specialists and the undesirable physico-chemical attributes of some vital substances like tretinoin and benzoyl peroxide (BPO) influence their usefulness and compliance of patient [14].

Topical therapy is the most well-known and mainstream approach to treat acne and there are a number of treatments options (Table 1) often in combination to have a synergistic effect and target simultaneous various pathogenic components. Generally, topical monotherapy is suggested for mild and moderate acne [22].

Table 1. Overview of topical antiacne agents $[14,16]$.

\begin{tabular}{|c|c|}
\hline Category & Drugs/Agents \\
\hline Topical bactericidals & BPO, triclosan, or chlorhexidine gluconate \\
\hline Topical antibiotics & Erythromycin, clindamycin, stievamycin, or tetracycline \\
\hline Hormonal treatments & Oestrogen/progestogen, antiandrogen, Cyproterone, in combination with an oestrogen \\
\hline Topical retinoids & Tretinoin, adapalene, and tazarotene, retinol \\
\hline Alpha or Beta Hydroxy acids & Glycolic acid, lactic acid, mandelic acid, lipohydroxy acid, salicylic acid \\
\hline Phototherapy & Nicitinamide, sodium ascorbyl phosphate, pyruvic acid, sulfur \\
\hline Miscellaneous & red light \\
\hline
\end{tabular}

Chiou (2012) evaluated the contribution of vehicle (placebo) toward the reduction in total (inflammatory and noninflammatory) lesion counts using $0.1 \%$ tretinoin, $0.1 \%$ adapalene, $5 \%$ dapsone, $1 \%$ clindamycin, a combination of benzoyl peroxide with adapalene or clindamycin, and a clindamycin-tretinoin combination and showed better significance of vehicle effects in topical therapy; in some cases this effect approached 90\% [23].

Recently in US patent no. 8846646, Chiou (2014) has reported an effective and safe method for treating acne and rosacea by topically applying a therapeutically effective amount of propylene glycol alone or in combination with a therapeutically effective amount of salicylic acid or other anti-acne or anti-rosacea compounds in a dosage form to the area of skin lesion [24].

There are different formulations available for the acne management, but most of them are lacking any scientificallyproven responses. Successful treatments show little improvement within the first two weeks, instead taking a period of approximately three months to improve and start flattening out [16].

Treatments are believed to work in at least 4 different ways (with many of the best treatments providing multiple simultaneous effects): normalizing shedding into the pore to prevent blockage, eradicating P. acnes, anti-inflammatory responses and hormonal control.

Combination therapies can significantly diminish the extent and severity of acne in many cases, but have greater potential for side effects and need a greater degree of monitoring. Table $\mathbf{1}$ indicates different topical anticane agents and Table 2 shows cutaneous side-effects from topical acne treatments and potential drug delivery systems for agent 
delivery.

Table 2. Cutaneous side effects from topical antiacne agents and potential drug delivery [22].

\begin{tabular}{|c|c|c|}
\hline Common Topical Acne Treatments & Cutaneous Side-effects & Drug Delivery \\
\hline $\begin{array}{c}\text { Retinoids (e.g., adapalene, } \\
\text { tazarotene, tretinoin) }\end{array}$ & Burning, peeling, erythema, dryness, photosensitivity & $\begin{array}{c}\text { Microsponges, liposomes, nanoemulsions, aerosol } \\
\text { foams }\end{array}$ \\
\hline BPO & Dryness, erythema, peeling, hair and clothing discoloration & Polymers, fullerenes \\
\hline Clindamycin phosphate & Erythema, dryness, allergic contact dermatitis & Aerosol foams, polymers, nanomemulsions \\
\hline Erythromycin & Dryness, erythema, peeling, allergic contact dermatitis & Aerosol foams, polymers, nanomemulsions \\
\hline Salicylic acid & Dryness, erythema, peeling & Polymers, microsponges \\
\hline
\end{tabular}

Another study by Lamel et al. (2015) revealed that clinical trial design, implementation, the biologic effects of vehicles, and natural disease progression influenced the patient responses in randomized controlled trials evaluating topical acne therapies for determination of efficacies of BPO [25].

\section{DRUG DELIVERY SYSTEMS}

Though topical treatment has an imperative role in acne treatment, side effects of different topical antiacne drugs and the unwanted physicochemical properties of certain important agents like tretinoin and BPO influence their use. Novel drug delivery can be used to improve the topical delivery of antiacne agents by promoting dermal localization and lowering their side effects. There are different advanced strategies like liposomes, niosomes, aspasomes microsponges, microemulsions, hydrogels and solid lipid nanoparticles to enhance the topical administration of antiacne agents [14].

\subsection{Liposomes}

Liposomes are often utilized as vehicles in pharmaceuticals and cosmetics for a modified drug delivery. They are considered to be spherical vesicles whose membrane comprises amphiphilic lipids that surround an aqueous core. Hydrophilic substances may be encapsulated in the aqueous core and lipophilic substances in the lipid bilayer so this enables the delivery of 2 types of substances once they are applied on the skin [26, 27].

The fundamental parts of liposomes are phospholipids (phosphatidylcholine, phophatidylethanolamine, phophatidylserine, dipalmitoyl phosphatidylcholine, and others), cholesterol, and water. Liposomes may differ considerably in terms of size and structure. One or more concentric bilayers emclose an aqueous core producing small or large unilamellar vesicles (SUV, LUV) or multilamellar vesicles (MLV), respectively [28, 29].

Liposomes are considered to be the first generation of novel drug delivery systems having diameters ranging from $80 \mathrm{~nm}$ to $100 \mu \mathrm{m} \mathrm{[14]}$. Due to their high degree of biocompatibility, liposomes have been used as advanced drug delivery systems for enclosing of molecules [28].

It was reported that Clindamycin hydrochloride multilamellar liposomes had been prepared using either lecithin and cholesterol or Hostaphat KW (Hoechst) and cholesterol acne management. In vitro diffusion studies on the Hostaphat liposomes exhibited a sustained release. Liposomal clindamycin lotion was much more effective in reducing the total number of comedones, papules and mainly pustules. Tretinoin, also known as all trans retinoic acid, is a successful antiacne drug used topically for reducing the size and number of comedones. Unfortunately, its poor aqueous solubility, photoliability, high instability in the presence of heat and light and skin irritating nature strongly limits its topical use. Moreover, tretinoin users experience side effects like erythema, peeling, burning at the application site and increased susceptibility to sunlight. In order to overcome these disadvantages and to improve its effectiveness after topical application, the use of liposomal formulations has been recommended [14].

Among liposomal formulations, positively charged liposomes exhibited enhanced tretinoin release and permeation as compared to negatively charged liposomes. Moreover, liposomal formulations improved tretinoin accumulation into newborn pig skin. Among liposomal formulations, the maximum accumulation values for tretinoin were found with negatively charged liposomes as compared to positively charged liposomes. Additionally, TEM analyses of the newborn pig skin-liposome interaction revealed that liposomes do not penetrate in the skin as intact structures and negatively charged liposomes strongly improved the hydration of pig skin. These reports suggested that the composition and charge of liposomes would play an important role in optimizing tretinoin delivery. Apart from the improved delivery, liposomal encapsulation of tretinoin is also expected to improve its photostability [28]. 
BPO is a successful topical agent in the treatment of acne. The key mechanism of action of BPO in acne is linked to its eradication property of $P$. acnes in the sebaceous follicle. Liposomal BPO gels exhibited a better stability profile with lower drug leakage as compared to BPO liposomal dispersions. The liposomal BPO gel showed a significant improvement in the therapeutic response (about 2-fold) at all times of evaluation when compared to the plain BPO gel $[14,30]$.

Combination therapy comprising of antiacne agents differing in mechanism of action is gaining importance in the effective management of acne. Topical retinoids combined with topical or oral antibiotics were reported to decrease acne lesions quicker and to a larger range than antimicrobial therapy alone [31].

Erythromycin and BPO have also been shown to possess synergism and can improve acne treatment. With this aim we have developed the combination gel containing liposome-encapsulated erythromycin and BPO. The in vitro skin permeation study indicated longer residence of erythromycin and BPO liposomes in the guinea pig skin [32].

The use of antiandrogen has received great attention in acne treatment ever since the sebaceous gland was found to be androgen sensitive. Cyproterone acetate (CPA) has been used since the early 1960s and denotes the first specific antiandrogen of clinical interest. It was reported that acne and hirsutism can be effectively managed with orally delivered CPA. However, oral administration of CPA is associated with side effects like lassitude, loss of libido, nausea and breast tenderness. Hence, a topical CPA formulation with the ability to deliver sufficient doses in order to target sebaceous glands with concomitant reduction in systemic CPA concentration would be an ideal approach in such a case. However, earlier trials conducted using topical CPA formulations were unsuccessful due to the lack of a suitable vehicle for transdermal delivery of CPA. Serum concentrations of CPA were ten-folds lesser after topical administration of CPA as compared to orally delivered CPA. Thus, liposomal CPA has opened a new avenue for effective acne treatment with reduced side effects [33].

Salicylic acid is widely used in the management of dry skin conditions and to minimize acne symptoms due to its keratolytic effect like sodium salycilate. However, users experience mild to strong irritation after application of salicylic acid. Since salicylic acid precipitates into a powder on the surface of the skin after solvent evaporation, it does not require neutralization. Hence, liposomal encapsulation can be an attractive approach for salicylic acid delivery. It was also shown that liposomal preparation of salicylic acid not only extended the salicylic acid release across the skin but also improved the retention of salicylic acid in the skin. Tea tree oil, a natural bioactive obtained from Melaleuca alternifolia, has also attained considerable interest in the topical treatment of acne due to its antibacterial properties. The study suggested that the liposomal approach would be a good option for improving the therapeutic efficacy of tea tree oil $[14,34,35]$.

\subsection{Niosomes}

Niosomes are bilayer structures obtained from amphiphiles in aqueous media. Different kinds of surfactants are employed for niosomes formulation. Principally, they are analogous to liposomes. Niosome formation necessitates the existence of specific amphiphiles like polyoxyethylene alkyl ethers, sorbitan esters, polysorbate-cholesterol mixtures, crown ether derivatives, perfluoroalkyl surfactants, alkyl glycerol ethers and others, and aqueous solvent [28].

Niosomes represent second-generation vesicular carriers which have evolved as a substitute to liposomes largely because of their higher chemical stability, enhanced encapsulation efficiency, intrinsic skin penetration-enhancing properties and lower cost of production as compared to liposomes. Various nonionic surfactants like polyoxyethylene alkyl ethers and esters, glucosyl dialkyl ethers, poly glycerol alkyl ethers, crown ethers and sorbitan esters can be utilized to prepare niosomes. Charge inducers like dicetyl phosphate are intercalated in bilayers to incorporate electrostatic repulsions among the vesicles for improving the stability of vesicles. However, charge inducers used in the formulation should be biocompatible. The classification of niosomes, their methods of preparation and advantages in the dermal delivery are similar as described under liposomes [36, 37].

Although the utility of niosomes in dermal delivery for the enhanced delivery of topical agents was realized around a decade ago, their potential in the antiacne drugs administration was not very well studied yet. The potential of niosomes has been explored for delivering tretinoin. Manconi et al. have recently reported the utility of niosomes for encapsulating tretinoin in order to improve its photochemical stability. They prepared multilamellar, large unilamellar and small unilamellar niosomes by using sorbitan esters, polyoxyethylene lauryl ether, and a commercial mixture of octyl/decyl polyglucosides in the presence of cholesterol and dicetyl phosphate. Properties of niosomes are influenced by the surfactant composition and interaction $[38,39]$. 
The photostability of tretinoin in either the 'free' or 'noisome-encapsulated' form was investigated by carrying out UV irradiation and artificial light irradiation. The integration of tretinoin in vesicles directed to a lessening of the photolysis of tretinoin and the photoprevention exhibited by the vesicles. Not all the studied vesicular formulations improved the stability of tretinoin in comparison with the stability of free tretinoin in methanol. Thus, niosomes can become interesting carriers for dermal delivery of antiacne agents and their ability needs to be explored to the fullest [40].

\subsection{Aspasomes}

Aspasomes are novel vesicular carriers recently investigated by Gopinath et al., which are formed by using ascorbyl palmitate, cholesterol and dicetyl phosphate. Aspasomes can gain importance in acne therapy as the potential of ascorbic acid derivatives in acne treatment has been realized. They can be fabricated by the typical film hydration method. Aspasomes have been characterized by conventional techniques for bilayer formation and thermal behavior by differential scanning calorimetry (DSC) analysis. Their advantages in dermal delivery of therapeutic agents are similar to that of liposomes. Moreover, their inherent antioxidant potential can be complementary to the topical acne therapy [40].

\subsection{Microsponges}

Microsponges are biologically inactive, non-irritating, non-mutagenic, non-allergic, non-toxic, and nonbiodegradable polymeric drug delivery systems, which exhibited great use in dermopharmaceuticals administration. Microsponges are also defined as porous microspheres, which are prepared by using cross-linked polymers like styrenedivinyl benzene by employing suitable polymerization techniques. Like a true sponge, a single Microsponge system comprises numerous interconnecting spaces within a noncollapsible structure with a large porous surface. These systems are produced mainly by suspension polymerization that leads to structures with an internal surface area ranging from 20 to $500 \mathrm{~m}^{2} / \mathrm{g}$. Moreover, the generation of Microsponges of varying size $(5-300 \mu \mathrm{m})$ is possible as the polymerization conditions can be varied over a wide range. A typical $25 \mu \mathrm{m}$ microsponge can have up to 250,000 pores, forming an entire pore volume of around $1 \mathrm{ml} / \mathrm{g}$. Furthermore, release from the microsponge could be modulated by using various triggers or stimuli like pressure, change in temperature, change in $\mathrm{pH}$, change in polarity and change in solubility. Microsponges mainly entrap the drug by sorption mechanisms [41].

The advantages that the microsponge offers in topical delivery are as follows: improved physical stability and better protection from environmental factors, reduction in side effects associated with the topical antiacne agents, sustained release of active agent thus prolonging the drug activity, ease of incorporation into formulations like gel, cream, liquid or powder, improved elegance and esthetic appeal, high drug payload, reduction in systemic absorption of the topical agents, and programmable and flexible delivery system since the release could be modulated by using various stimuli so as to tailor the release of active moiety on command. Microsponge technology has been successfully applied for improving tolerability of antiacne agents like BPO and tretinoin. Furthermore, tretinoin microsponges have reached commercialization and are currently marketed as Retin-A Micro ${ }^{\circledR}$. Retin-A Micro consists of microsponges (based on methyl methacrylate/glycol dimethacrylate crosspolymers) of tretinoin incorporated in an aqueous gel. It is available in two strengths, namely 0.1 and $0.04 \%$ [14].

\subsection{Emulsifier-free Formulations}

They are also expanding for delivery of cosmetic and dermatologic formulations. Most skin care products are emulsions so during production they require the addition of surfactants ("emulsifiers"). Additionally, when the surfactant agents are spread on the skin, they emulsify and get rid of the natural lipids. Thus, the pharmaceutical factory is evolving them as substitutes to conventional preparations [22].

\subsection{Microemulsions}

Microemulsions are thermodynamically stable, isotropic, and transparent, low-viscosity colloidal dispersions comprising micro-domains of oil and/or water stabilized by an interfacial film of alternating surfactant and cosurfactant molecules. They contain swollen micellar (oil-in-water, $\mathrm{O} / \mathrm{W}$ ), reverse micellar (water-in-oil, W/O) and bicontinuous structures.

The advent of microemulsion-based gels (which amalgamate the advantages of microemulsions and gels) has helped in improving the patient compliance of microemulsions. Microemulsion-based gels are produced by using a suitable 
polymer that is capable of modifying the rheological behavior of microemulsions. However, it is essential to ensure that the polymer does not alter the desirable features of microemulsions. Several gelling agents have already been studied for their potential to form microemulsion-based gels [14].

Azelaic acid, a bioactive molecule utilized in managing acne and different skin complications, was first formulated in the form of microemulsions. Gasco et al. successfully achieved incorporation of azelaic acid into the microemulsion based on topically acceptable components like propylene glycol, decanol, dodecanol and polysorbate 20 and microemulsions were viscosized using Carbopol. An investigation of the in vitro diffusion profile of azelaic acid from marketed gel and microemulsion-based gel revealed that the microemulsion-based gels dramatically improved the transport of the azelaic acid across hairless mouse skin. Additionally, the effect of penetration enhancer like DMSO was studied on the permeation of azelaic acid from microemulsion-based gels. The permeation of azelaic acid increased with an increase in the DMSO content [14, 42].

\subsection{Fullerenes}

They are compounds made entirely of carbon that look like a hollow sphere. It was reported that when fullerenes come into touch with the skin, they migrate across the skin intercellularly. Hence, a fullerene could be employed to "trap" active substances and then release them into the epidermis upon skin application. Furthermore, fullerenes are considered to be possibly powerful antioxidants [22].

\subsection{Hydrogels /Polymers}

Hydrogels denote a novel drug delivery system comprising different applications. Their potential in peroral drug delivery and tissue engineering has been very well established and their potential in delivery of dermopharmaceuticals has been realized. Moreover, their biocompatibility and structural diversity have extended their utility for an array of applications. They are hydrophilic polymer linkages that exhibit the capacity of water absoprtion ranging from 10-20\% up to thousands of times their dry weight in water [43].

Hydrogels produced in the form of sheets can appropriately be utilized in the dermal or transdermal administration of the active constituents. Moreover, by modulating the composition of the polymeric backbone and reaction conditions (in case of cross-linked hydrogels) hydrogels can be tailored to obtain desired physical properties and release profile. Owing to their versatility, hydrogels can be easily employed for localized delivery of antiacne agents especially the ones exhibiting the problem of skin irritation. In fact, the hydrogels exhibiting notable skin adhesion and moisturizer effect would be an ideal tactic for topical acne treatment.

Lee et al. [44] have successfully applied 'hydrogel technology' for improving dermal accumulation of an antiacne agent in order to avoid their percutaneous absorption. The investigation has not only revealed the potential of triclosan as an antiacne agent but also provided the platform technology for efficient topical delivery of various other antiacne agents. Lee et al. have designed peeloff-type adhesive hydrogel patches based on 2 matrix polymers, namely sodium polyacrylate and sodium carboxymethyl cellulose. The cross-linking of these negatively charged polymers was achieved by employing $\mathrm{Al}^{3+}$ ion generated in situ. They found that in order to obtain hydrogel in the patch form, the cross-linking reaction should be slow and the solidification of the hydrogel should not be completed before casting the fluidic gel as a thin film.

Hydrogel patches containing triclosan in varying concentrations (from 0.01 to $0.5 \% \mathrm{w} / \mathrm{w}$ ) were characterized for $i n$ vitro antimicrobial activity against $P$. acnes (ATCC 6919), adhesivity and in vitro skin permeation. The antimicrobial activity of triclosan hydrogel patches was evident when the triclosan content was $0.05 \% \mathrm{w} / \mathrm{w}$ and it improved with the escalation in the amount of triclosan. In vitro permeation studies carried out on triclosan hydrogel patches using hairless mouse skin clearly indicated the significant increase in the amount of triclosan transported into the skin as compared to the amount transported across the skin [45].

\subsection{Solid Lipid Nanoparticles}

Solid lipid nanoparticles (SLN TM, Lipoearls TM) are advanced drug administration systems that are introduced for combining the advantages but avoiding the disadvantages of conventional colloidal drug carrier systems like polymeric nanoparticles, liposomes and microparticles and emulsions. They have been proved to be promising delivery strategy for efficient administration of many active constituents by numerous application pathways. SLNs have revealed a great ability to efficiently deliver various topical agents and even cosmeceuticals and their applications are continuously 
being unraveled. It was reported the successful fabrication of SLNs for improved dermal delivery of tretinoin using the novel solvent emulsification diffusion method. Interestingly, SLN-based tretinoin gels were greatly able to improve the tolerability of tretinoin as compared to commercialized products when valued by the Draize patch test in rabbits [14, $45]$.

\subsection{Aerosol Foams}

They have become an exceedingly common kind of topical preparation for a diversity of skin disorders like acne. The vehicle base of the foam could possess a liquid or semi-solid consistency that contains the same physicochemical properties of conventional vehicles like creams, lotions and gels, but it balances desirable characterstics such as moisturizing/fast-drying effects, or higher drug bioavailability. The aerosol base is dispensed through a gas-pressurized can that discharges the foam. The product features are determined by the type of formulation and the dispensing container that are selected to suit the specific therapy needs. In acne, foams may be preferred for application on large hairy surfaces or on the face as cleansers, because they are easier to spread [22].

Table 3 shows the summary of novel drug delivery systems for antiacne agents.

Table 3. Novel drug delivery systems for antiacne agents $[14,16,22,28,45,46]$.

\begin{tabular}{|c|c|c|c|}
\hline $\begin{array}{l}\text { Drug delivery } \\
\text { systems }\end{array}$ & Drug enclosed & Limitations with conventional therapy & Merits \\
\hline \multirow{4}{*}{ Nanoparticles } & Minocycline & $\begin{array}{c}\text { Lack of drug loading and entrapment efficiency } \\
\text { due to hydrophilicity of the drug }\end{array}$ & $\begin{array}{c}\text { Enhanced drug loading and entrapment efficiency and } \\
\text { controlled release }\end{array}$ \\
\hline & Azelaic acid & Fewer side effects & Enhanced drug retention at PSU and stability \\
\hline & Triclosan & $\begin{array}{l}\text { Insufficient permeation and absorption via } \\
\text { cutaneous route }\end{array}$ & Non-irritant to skin, enhanced stability \\
\hline & CPA & Systemic antiandrogenic effects & Increased skin penetration and absorption \\
\hline Niosomes & Tretinoin & Photodegradation & $\begin{array}{c}\text { Increased accumulation in superficial stratum and } \\
\text { stability, Increased drug release and entrapment } \\
\text { efficiencies }\end{array}$ \\
\hline \multirow{5}{*}{ Liposomes } & Isotretinoin & $\begin{array}{c}\text { Skin irritation, very low water solubility, } \\
\text { difficulty to incorporate in topical base, } \\
\text { photodegradation }\end{array}$ & $\begin{array}{l}\text { Potential for skin targeting, prolonging drug release, } \\
\text { reduction of photodegradation and skin irritation }\end{array}$ \\
\hline & $\begin{array}{l}\text { Clindamycin } \\
\text { hydrochloride }\end{array}$ & Lesser reduction in number of lesions & $\begin{array}{l}\text { Enhanced antiacne activity and sustained release of } \\
\text { drug }\end{array}$ \\
\hline & Tretinoin & Skin irritant, photo instability & $\begin{array}{c}\text { Enhanced local tolerability and 5-6 times increase } \\
\text { comedolytic activity, } \\
\text { Reduced photo instability }\end{array}$ \\
\hline & Salicylic acid & Skin irritation & Increased entrapment efficiency and stability \\
\hline & $\mathrm{BPO}$ & Skin irritation & Improved antibacterial activity, reduced irritation \\
\hline \multirow{4}{*}{$\begin{array}{c}\text { Solid lipid } \\
\text { nanoparticles }\end{array}$} & Isotretinoin & $\begin{array}{l}\text { Teratogenicity, mucocutaneous problems like } \\
\text { cheilitis, dermatitis, conjunctivitis, blepharitis, } \\
\text { skin fragility and xerosis, psychological } \\
\text { disorders, erythema, dryness, itching, stinging, } \\
\text { skin peeling }\end{array}$ & $\begin{array}{l}\text { Reduced dermal irritation, increased therapeutic } \\
\text { performance }\end{array}$ \\
\hline & Retinoic acid & $\begin{array}{l}\text { Sensitive to sunlight, eczematous irritation, } \\
\text { erythema, interaction with other applied } \\
\text { products }\end{array}$ & Comedolytic effect, reduction in RA induced irritation \\
\hline & Tretinoin & Skin irritation and chemical instability & $\begin{array}{l}\text { High encapsulation efficacy, physical stability and } \\
\text { absence of cytotoxicity }\end{array}$ \\
\hline & $\begin{array}{c}\text { Terbinafine } \\
\text { hydrochloride }\end{array}$ & Longer duration of treatment & Controlled release, drug targeting \\
\hline Nanosuspension & Tretinoin & Poor water solubility and photostability & $\begin{array}{l}\text { Improved drug permeation and UV irradiation } \\
\text { stability }\end{array}$ \\
\hline Nanoemulsion & $\begin{array}{l}\text { Tretinoin, } \\
\text { Tetracycline }\end{array}$ & Skin irritation, a burning sensation, and peeling & Enhanced drug permeation and antibacterial activity \\
\hline Nano lipid carriers & $\begin{array}{l}\text { Tretinoin, } \\
\text { Tetracycline }\end{array}$ & Skin irritation, a burning sensation, and peeling & Enhanced drug permeation and antibacterial activity \\
\hline
\end{tabular}




\begin{tabular}{|c|c|c|c|}
\hline $\begin{array}{c}\text { (Table } ₫ \text { ) contd..... } \\
\text { Drug delivery } \\
\text { systems }\end{array}$ & Drug enclosed & Limitations with conventional therapy & Merits \\
\hline \multirow{4}{*}{ Microemulsions } & Azelaic acid & Large and frequent dosing & Enhanced stability \\
\cline { 2 - 4 } & Tretinoin & systemic side effects & $\begin{array}{c}\text { Reducing frequency of administration, potentially } \\
\text { decreasing side effects, improved patient compliance } \\
\text { sustaining drug delivery }\end{array}$ \\
\cline { 2 - 4 } & Retinoic acid & Systemic side effects & Enhanced skin accumulation of retinoic acid \\
\hline \multirow{3}{*}{ Microspheres } & BPO & Skin irritation & $\begin{array}{c}\text { Appropriate reduction in P. acnes count, reduced skin } \\
\text { irritation }\end{array}$ \\
\cline { 2 - 4 } & Retinoid & Skin irritation and instability & Reduced irritation and enhanced stability \\
\hline Hydrogels & Triclosan & $\begin{array}{c}\text { Insufficient permeation and absorption via } \\
\text { cutaneous route }\end{array}$ & Enhanced transdermal penetration \\
\hline
\end{tabular}

\section{CONCLUSION}

Novel drug delivery systems display a strong base for topical management of acne in order to enhance the therapeutic performance of the topical antiacne agents with improved patience compliance and a concomitant reduction in their side effects. Amongst various drug delivery systems: liposomes have been extensively explored and their ability to optimize and improve topical therapy has been proved by several clinical trials. However, the potential of various other vesicular carriers like niosomes, aspasomes, ethosomes and transferosomes still remains to be fully investigated. Microemulsions, microsponges, solid lipid nanoparticles and hydrogels also exhibit a tremendous potential for commercialization. In fact, their potential for successful topical delivery of antiacne agents has been very well reported by in vitro experiments.

\section{CONFLICT OF INTEREST}

The author confirms that this article content has no conflict of interest.

\section{ACKNOWLEDGEMENTS}

Declared none.

\section{REFERENCES}

[1] Miranda W, Maja P, Joke AB. The lipid organization in SC and model systems base on ceramides. In: Enhancement in drug delivery. USA: CRS Press Taylor \& Francis Group 2007; pp. 217-20.

[2] Blank IH. Transport across the SC. Toxicol Appl Pharmacol 1969; 14(Suppl. 3): 23-9. [http://dx.doi.org/10.1016/S0041-008X(69)80006-2]

[3] Scheuplein RJ, Blank IH. Permeability of the skin. Physiol Rev 1971; 51(4): 702-47. [PMID: 4940637]

[4] Eckert RL. Structure, function, and differentiation of the keratinocyte. Physiol Rev 1989; 69(4): 1316-46. [PMID: 2678169]

[5] Baker H, Kligman AM. Technique for estimating turnover time of human stratum corneum. Arch Dermatol 1967; 95(4): 408-11. [http://dx.doi.org/10.1001/archderm.1967.01600340068016] [PMID: 4164581]

[6] Bouwstra JA, de Graaff A, Gooris GS, Nijsse J, Wiechers JW, van Aelst AC. Water distribution and related morphology in human stratum corneum at different hydration levels. J Invest Dermatol 2003; 120(5): 750-8. [http://dx.doi.org/10.1046/j.1523-1747.2003.12128.x] [PMID: 12713576]

[7] Elias PM. Epidermal lipids, barrier function, and desquamation. J Invest Dermatol 1983; 80: 44. [http://dx.doi.org/10.1038/jid.1983.12] [PMID: 6184422]

[8] Barry BW. Structure, function, diseases, and topical treatment of human skin. In: Dermatological formulations percutaneous absorption, 1. New York: Marcel Dekker 1983.

[9] Rolland A, Wagner N, Chatelus A, Shroot B, Schaefer H. Site-specific drug delivery to pilosebaceous structures using polymeric microspheres. Pharm Res 1993; 10(12): 1738-44. [http://dx.doi.org/10.1023/A:1018922114398] [PMID: 8302759]

[10] Grams YY, Alaruikka S, Lashley L, Caussin J, Whitehead L, Bouwstra JA. Permeant lipophilicity and vehicle composition influence accumulation of dyes in hair follicles of human skin. Eur J Pharm Sci 2003; 18(5): 329-36. [http://dx.doi.org/10.1016/S0928-0987(03)00035-6] [PMID: 12694885]

[11] Cullander C, Guy RH. Visualization of iontophoretic pathways with confocal microscopy and the vibrating probe electrode. Solid State Ion 1992; 53-56: 197.

[http://dx.doi.org/10.1016/0167-2738(92)90382-Y] 
[12] Williams ML, Elias PM. The extracellular matrix of stratum corneum: role of lipids in normal and pathological function. Crit Rev Ther Drug Carrier Syst 1987; 3(2): 95-122. [PMID: 3542246]

[13] Fries JH. Chocolate: a review of published reports of allergic and other deleterious effects, real or presumed. Ann Allergy 1978; 41(4): 195-207. [PMID: 152075]

[14] Date AA, Naik B, Nagarsenker MS. Novel drug delivery systems: potential in improving topical delivery of antiacne agents. Skin Pharmacol Physiol 2006; 19(1): 2-16. [http://dx.doi.org/10.1159/000089138] [PMID: 16247244]

[15] Ballangera F, Baudrya P, N'Guyenb JM, Khammaria A, Dréno B. Heredity: a prognostic factor for acne. Dermatology 2005; $212(2)$ : 145.: 9.

[16] Dermatological Services - Acne. Available from: http://www.adultandpediatricdermatology.com/acne.shtml [Accessed on June 20, 2016].

[17] Simpson NB, Cunliffe WJ. Disorders of the sebaceous glands. In: Burns T, Ed. Rook's textbook of dermatology. $7^{\text {th }}$ ed. Malden, Mass.: Blackwell Science 2004; pp. 43-75.

[http://dx.doi.org/10.1002/9780470750520.ch43]

[18] Adebamowo CA, Spiegelman D, Berkey CS, et al. Milk consumption and acne in adolescent girls. Dermatol Online J 2006; 12 (4): 1. [PMID: 17083856]

[19] Arbesman H. Dairy and acnethe iodine connection. J Am Acad Dermatol 2005; 53(6): 1102. [http://dx.doi.org/10.1016/j.jaad.2005.05.046] [PMID: 16310091]

[20] Fulton JE Jr, Plewig G, Kligman AM. Effect of chocolate on acne vulgaris. JAMA 1969; 210(11): 2071-4. [http://dx.doi.org/10.1001/jama.1969.03160370055011] [PMID: 4243053]

[21] Acne Scars - The Physical Effects of Acne. Available from: http:/www.proactiv.com.au/physical-effects-of- acne.aspx [Accessed Date: June 20, 2016].

[22] Taglietti M, Hawkins CN, Rao J. Novel topical drug delivery systems and their potential use in acne vulgaris. Skin Therapy Lett 2008; 13(5): 6-8. [PMID: 18648713]

[23] Chiou WL. Low intrinsic drug activity and dominant vehicle (placebo) effect in the topical treatment of acne vulgaris. Int J Clin Pharmacol Ther 2012; 50(6): 434-7.

[http://dx.doi.org/10.5414/CP201694] [PMID: 22677304]

[24] Chiou WL. Topical treatment of skin infection. US Patent US8846646 B2, 2014.

[25] Lamel SA, Sivamani RK, Rahvar M, Maibach HI. Evaluating clinical trial design: systematic review of randomized vehicle-controlled trials for determining efficacy of benzoyl peroxide topical therapy for acne. Arch Dermatol Res 2015; 307(9): 757-66. [http://dx.doi.org/10.1007/s00403-015-1568-9] [PMID: 26048131]

[26] Schäfer-Korting M, Korting HC, Ponce-Pöschl E. Liposomal tretinoin for uncomplicated acne vulgaris. Clin Investig 1994; 72(12): 1086-91. [http://dx.doi.org/10.1007/BF00577761] [PMID: 7711421]

[27] Brisaert M, Gabriëls M, Matthijs V, Plaizier-Vercammen J. Liposomes with tretinoin: a physical and chemical evaluation. J Pharm Biomed Anal 2001; 26(5-6): 909-17.

[http://dx.doi.org/10.1016/S0731-7085(01)00502-7] [PMID: 11600303]

[28] Elka T, Biana G. Vesicular carriers enhanced delivery through the skin. In: Enhancement in drug delivery. USA: CRS Press Taylor \& Francis Group 2007; pp. 255-63.

[29] Peter AM, Kathleen MB. Lipids of Physiologic Significance. In: Harper's Illustrated Biochemistry. $6^{\text {th }}$ Edition. USA: McGraw-Hill Companies, Inc. 2003; p. 120.

[30] Patel VB, Misra AN, Marfatia YS. Preparation and comparative clinical evaluation of liposomal gel of benzoyl peroxide for acne. Drug Dev Ind Pharm 2001; 27(8): 863-9.

[http://dx.doi.org/10.1081/DDC-100107251] [PMID: 11699839]

[31] Gollnick H, Cunliffe W, Berson D, et al. Management of acne: a report from a global alliance to improve outcomes in acne. J Am Acad Dermatol 2003; 49(1)(Suppl.): S1-37. [http://dx.doi.org/10.1067/mjd.2003.618] [PMID: 12833004]

[32] Burkhart CN, Specht K, Neckers D. Synergistic activity of benzoyl peroxide and erythromycin. Skin Pharmacol Appl Skin Physiol 2000; 13(5): 292-6.

[http://dx.doi.org/10.1159/000029936] [PMID: 10940820]

[33] Gruber DM, Sator MO, Joura EA, Kokoschka EM, Heinze G, Huber JC. Topical cyproterone acetate treatment in women with acne: a placebo-controlled trial. Arch Dermatol 1998; 134(4): 459-63. [http://dx.doi.org/10.1001/archderm.134.4.459] [PMID: 9554298]

[34] Eremia S. Chemical Peels and Microdermabrasion. In: Office-Based Cosmetic Procedures and Techniques. $1^{\text {st }}$ ed. UK: Cambridge University Press 2010; pp. 338-42. [http://dx.doi.org/10.1017/CBO9780511674839] 
[35] Small R, Hoang D, Linder J. Chemical Peels. In: A Practical Guide to Chemical Peels, Microdermabrasion \& Topical Products. USA: Lippincott Williams \& Wilkins 2013; pp. 43-5.

[36] Namdeo A, Jain NK. Niosomes as drug carriers. Indian J Pharm Sci 1996; 58: 41-6.

[37] Uchegbu IF, Vyas SP. Non-ionic surfactant based vesicles (niosomes) in drug delivery. Int J Pharm 1998; 172: 33-70. [http://dx.doi.org/10.1016/S0378-5173(98)00169-0]

[38] Manconi M, Sinico C, Valenti D, Loy G, Fadda AM, Fadda AM. Niosomes as carriers for tretinoin. I. Preparation and properties. Int J Pharm 2002; 234(1-2): 237-48 [http://dx.doi.org/10.1016/S0378-5173(01)00971-1] [PMID: 11839454]

[39] Manconi M, Valenti D, Sinico C, Lai F, Loy G, Fadda AM. Niosomes as carriers for tretinoin. II. Influence of vesicular incorporation on tretinoin photostability. Int J Pharm 2003; 260(2): 261-72. [http://dx.doi.org/10.1016/S0378-5173(03)00268-0] [PMID: 12842345]

[40] Gopinath D, Ravi D, Rao BR, Apte SS, Renuka D, Rambhau D. Ascorbyl palmitate vesicles (Aspasomes): formation, characterization and applications. Int J Pharm 2004; 271(1-2): 95-113. [http://dx.doi.org/10.1016/j.ijpharm.2003.10.032] [PMID: 15129977]

[41] Embil K, Nacht S. Microsponge delivery systems (MDS) a topical delivery system with reduced irritancy incorporating multiple mechanisms for triggering the release of active agents. J Microencapsul 1996; 13: 575-88 [http://dx.doi.org/10.3109/02652049609026042] [PMID: 8864994]

[42] Gasco MR, Gallarate M, Pattarino F. In vitro permeation of azelaic acid from microemulsions. Int J Pharm 1991; 69: 193-6. [http://dx.doi.org/10.1016/0378-5173(91)90361-Q]

[43] Hoffman AS. Hydrogels for biomedical applications. Adv Drug Deliv Rev 2002; 54(1): 3-12. [http://dx.doi.org/10.1016/S0169-409X(01)00239-3] [PMID: 11755703]

[44] Lee TW, Kim JC, Hwang SJ. Hydrogel patches containing triclosan for acne treatment. Eur J Pharm Biopharm 2003; 56(3): 407-12. [http://dx.doi.org/10.1016/S0939-6411(03)00137-1] [PMID: 14602184]

[45] Sinha P, Srivastava S, Mishra N, Yadav NP. New perspectives on antiacne plant drugs: contribution to modern therapeutics. BioMed Res Int 2014; 2014: 301304. [http://dx.doi.org/10.1155/2014/301304] [PMID: 25147793]

[46] Mortazavi SA, Pishrochi S, Jafari Azar Z. Formulation and in vitro evaluation of tretinoin microemulsion as a potential carrier for dermal drug delivery. Iran J Pharm Res 2013; 12(4): 599-609. [PMID: 24523740]

(C) Tesfaye Gabriel; Licensee Bentham Open

This is an open access article licensed under the terms of the Creative Commons Attribution-Non-Commercial 4.0 International Public License (CC BY-NC 4.0) (https://creativecommons.org/licenses/by-nc/4.0/legalcode), which permits unrestricted, non-commercial use, distribution and reproduction in any medium, provided the work is properly cited. 